\title{
COMPLETE LIFTING OF DOUBLE-LINEAR SEMI-BASIC TANGENT VALUED FORMS TO WEIL LIKE FUNCTORS ON DOUBLE VECTOR BUNDLES
}

\author{
WŁODZIMIERZ M. MIKULSKI
}

\begin{abstract}
Let $F$ be a product preserving gauge bundle functor on double vector bundles. We introduce the complete lifting $\mathcal{F} \varphi: F K \rightarrow \wedge^{p} T^{*} F M \otimes$ $T F K$ of a double-linear semi-basic tangent valued $p$-form $\varphi: K \rightarrow \wedge^{p} T^{*} M \otimes$ $T K$ on a double vector bundle $K$ with base $M$. We prove that this complete lifting preserves the Frolicher-Nijenhuis bracket. We apply the results obtained to double-linear connections.
\end{abstract}

\section{INTRODUCTION}

We assume that any manifold considered in the paper is Hausdorff, second countable, finite dimensional, without boundary and smooth (i.e. of class $C^{\infty}$ ). All maps between manifolds are assumed to be smooth (of class $C^{\infty}$ ).

Definition 1.1. An almost double vector bundle is a system $K=\left(K_{r}, K_{l}, E_{r}, E_{l}\right)$ of vector bundles $K_{r}=\left(K, \tau_{r}, E_{r}\right), K_{l}=\left(K, \tau_{l}, E_{l}\right), E_{r}=\left(E_{r}, \underline{\tau}_{l}, M\right)$ and $E_{l}=$ $\left(E_{l}, \underline{\tau}_{r}, M\right)$ such that $\underline{\tau}_{l} \circ \tau_{r}=\underline{\tau}_{r} \circ \tau_{l}$ (this means that the respective diagram is commutative). We call $M$ the basis of $K$.

If $K^{\prime}=\left(K_{r}^{\prime}, K_{l}^{\prime}, E_{r}^{\prime}, E_{l}^{\prime}\right)$ is another almost double vector bundle, an almost double vector bundle map $K \rightarrow K^{\prime}$ is a map $f: K \rightarrow K^{\prime}$ such that there are maps $\underline{f}_{r}: E_{r} \rightarrow E_{r}^{\prime}, \underline{f}_{l}: E_{l} \rightarrow E_{l}^{\prime}$ and $\underline{f}: M \rightarrow M^{\prime}$ such that $\left(f, \underline{f}_{r}\right): K_{r} \rightarrow K_{r}^{\prime}$, $\left(f, \underline{f}_{l}\right): K_{l} \rightarrow K_{l}^{\prime},\left(\underline{f}_{r}, \underline{f}\right): E_{r} \rightarrow E_{r}^{\prime}$ and $\left(\underline{f}_{l}, \underline{f}\right): E_{l} \rightarrow E_{l}^{\prime}$ are vector bundle maps. We call $f: M \rightarrow \bar{M}^{\prime}$ the base map of $f$.

For example, we have the trivial almost double vector bundle $K=\left(K_{r}, K_{l}, E_{r}, E_{l}\right)$, where $K_{l}=\left(\mathbf{R}^{m_{1}} \times \mathbf{R}^{m_{2}} \times \mathbf{R}^{n_{1}} \times \mathbf{R}^{n_{2}}, \tau_{l}, \mathbf{R}^{m_{1}} \times \mathbf{R}^{n_{1}}\right), K_{r}=\left(\mathbf{R}^{m_{1}} \times \mathbf{R}^{m_{2}} \times \mathbf{R}^{n_{1}} \times\right.$ $\left.\mathbf{R}^{n_{2}}, \tau_{r}, \mathbf{R}^{m_{1}} \times \mathbf{R}^{m_{2}}\right), E_{r}=\left(\mathbf{R}^{m_{1}} \times \mathbf{R}^{m_{2}}, \underline{\tau}_{l}, \mathbf{R}^{m_{1}}\right)$ and $E_{l}=\left(\mathbf{R}^{m_{1}} \times \mathbf{R}^{n_{1}}, \underline{\tau}_{r}, \mathbf{R}^{m_{1}}\right)$, and where $\tau_{r}, \tau_{l}, \underline{\tau}_{r}, \underline{\tau}_{l}$ are the obvious projections. We will denote this trivial almost double vector bundle by $\mathbf{R}^{m_{1}, m_{2}, n_{1}, n_{2}}$.

Definition 1.2. A double vector bundle is a locally trivial almost double vector bundle $K$. This means that there are nonnegative integers $m_{1}, m_{2}, n_{1}, n_{2}$ such

2020 Mathematics Subject Classification. 58A20, 53A55.

Key words and phrases. double vector bundle, double-linear semi-basic tangent valued $p$-form, complete lifting, product preserving gauge bundle functor. 
that for any $x \in M$ there is an open neighborhood $\Omega \subset M$ of $x$ such that $K_{\mid \Omega}=$ $\mathbf{R}^{m_{1}, m_{2}, n_{1}, n_{2}}$ modulo an almost double vector bundle isomorphism.

The tangent bundle

$$
T E=\left(\left(T E, \pi^{T E}, E\right),(T E, T \pi, T M),(E, \pi, M),\left(T M, \pi^{T M}, M\right)\right)
$$

of a vector bundle $E=(E, \pi, M)$ is an example of a double vector bundle.

Any manifold $M$ can be treated as the double vector bundle $M$ with basis $M$.

Definition 1.3. Let $K$ be a double vector bundle as above. A double-linear vector field on $K$ is a vector field $Z$ on $K$ such that the flow of $Z$ is formed by (local) double vector bundle isomorphisms.

Any double linear vector field $Z$ on $K$ is projectable with respect to the (common) projection $K \rightarrow M$. Thus we have the underlying vector field $\underline{Z}$ on $M$.

Definition 1.4. Let $K$ be a double vector bundle as above with basis $M$. A doublelinear semi-basic tangent valued $p$-form on $K$ is a section $\varphi: K \rightarrow \wedge^{p} T^{*} M \otimes T K$ such that $\varphi\left(X_{1}, \ldots, X_{p}\right)$ is a double linear vector field on $K$ for any vector fields $X_{1}, \ldots, X_{p}$ on the basis $M$ of $K$.

Definition 1.5. Let $K$ be as above. A double-linear connection in $K$ is a doublelinear semi-basic tangent valued 1-form $\Gamma: K \rightarrow T^{*} M \otimes T K$ on $K$ such that the underlying vector field of $\Gamma(X)$ is equal to $X$ for any vector field $X$ on basis $M$.

Let $\mathcal{D} \mathcal{V B}$ denote the category of all double vector bundles and their almost double vector bundle maps, and let $\mathcal{F M}$ denote the category of fibered manifolds and fibered maps. (In [14], the notation $2-\mathcal{V B}$ instead of $\mathcal{D V} \mathcal{B}$ is used.)

The general concept of (gauge) bundle functors can be found in [7]. We need the following particular case of it.

Definition 1.6. A gauge bundle functor on $\mathcal{D} \mathcal{V B}$ is a covariant functor $F: \mathcal{D} \mathcal{V B} \rightarrow$ $\mathcal{F M}$ sending any double vector bundle $K$ with basis $M$ into a fibered manifold $p_{K}: F K \rightarrow M$ over $M$ and any double vector bundle map $f: K \rightarrow K^{\prime}$ with the base map $\underline{f}: M \rightarrow M^{\prime}$ into a fibered map $F f: F K \rightarrow F K^{\prime}$ over $\underline{f}: M \rightarrow M^{\prime}$, and satisfying the following conditions:

(i) Localization condition: For every double vector bundle $K$ with basis $M$ and any open subset $U \subset M$ the inclusion map $i_{K \mid U}: K \mid U \rightarrow K$ induces a diffeomorphism $F i_{K \mid U}: F(K \mid U) \rightarrow p_{K}^{-1}(U)$.

(ii) Regularity condition: $F$ transforms smoothly parametrized families of $\mathcal{D} \mathcal{V B}$ maps into smoothly parametrized families of $\mathcal{F} \mathcal{M}$-maps.

A gauge bundle functor $F$ on $\mathcal{D} \mathcal{V B}$ is product preserving (ppgb-functor) if $F\left(K_{1} \times K_{2}\right)=F\left(K_{1}\right) \times F\left(K_{2}\right)$ for any $\mathcal{D} \mathcal{V B}$-objects $K_{1}$ and $K_{2}$. Product preserving gauge bundle functors can be also called Weil like functors, because the product preserving bundle functors on manifolds are the usual Weil functors.

A simple example of a ppgb-functor on $\mathcal{D V B}$ is the tangent functor $T$ sending any $\mathcal{D} \mathcal{V B}$-object $K$ into the tangent bundle $T K$ (over $M$ ) and any $\mathcal{D} \mathcal{V B}$-map $f: K \rightarrow K^{\prime}$ into the tangent map $T f: T K \rightarrow T K^{\prime}$. 
By 14, the ppgb-functors $F$ on $\mathcal{D} \mathcal{V B}$ are in bijection with the $A^{F}$-bilinear maps $\diamond^{F}: U^{F} \times V^{F} \rightarrow W^{F}$, where $A^{F}$ are Weil algebras and $U^{F}, V^{F}$ and $W^{F}$ are finitely dimensional (over $\mathbf{R}$ ) $A^{F}$-modules. Moreover, the ppgb-functors $F$ on $\mathcal{D} \mathcal{V B}$ have values in $\mathcal{D} \mathcal{V B}$. For any such $F$, if $K$ is a $\mathcal{D} \mathcal{V B}$-object with basis $M$, then $F K$ is a $\mathcal{D} \mathcal{V B}$-object with basis $F M=T^{A^{F}} M$; see [14.

Let $F$ be a ppgb-functor on $\mathcal{D} \mathcal{V B}$ and let $\diamond^{F}: U^{F} \times V^{F} \rightarrow W^{F}$ be the corresponding $A^{F}$-bilinear map. Let $K$ be a $\mathcal{D} \mathcal{V B}$-object. Then any doublelinear vector field $Z$ on $K$ can be lifted to the double-linear vector field $\mathcal{F} Z$ on $F K$ via $F$-prolongation of flow. By [14, for any $a \in A^{F}$ we have the affinor af $(a): T F K \rightarrow T F K$ on $F K$. We have af $\left(a_{1} a_{2}\right)=\operatorname{af}\left(a_{1}\right) \circ$ af $\left(a_{2}\right)$ and af(1) is the identity affinor. If $f: K \rightarrow K_{1}$ is a $\mathcal{D} \mathcal{V B}$-map, then $T F f \circ \operatorname{af}(a)=\operatorname{af}(a) \circ T F f$.

The main result of the paper is the following one (see Theorem 4.5):

Let $F$ be a ppgb-functor on $\mathcal{D} \mathcal{V B}$. Let $\varphi: K \rightarrow \wedge^{p} T^{*} M \otimes T K$ be a doublelinear semi-basic tangent valued $p$-form on a double vector bundle $K$ with basis $M$. Then there exists one and only one double-linear semi-basic tangent valued $p$-form $\mathcal{F} \varphi: F K \rightarrow \wedge^{p} T^{*} F M \otimes T F K$ on $F K$ such that

$$
\mathcal{F} \varphi\left(\operatorname{af}\left(a_{1}\right) \circ \mathcal{F} X_{1}, \ldots, \operatorname{af}\left(a_{p}\right) \circ \mathcal{F} X_{p}\right)=\operatorname{af}\left(a_{1} \cdot \ldots \cdot a_{p}\right) \circ \mathcal{F}\left(\varphi\left(X_{1}, \ldots, X_{p}\right)\right)
$$

for any vector fields $X_{1}, \ldots, X_{p}$ on $M$ and any $a_{1}, \ldots, a_{p} \in A^{F}$.

Definition 1.7. We call $\mathcal{F} \varphi$ (as above) the complete lift of $\varphi$ to $F$.

Next we study the complete lifting $\mathcal{F}$. We prove that $\mathcal{F}$ commutes with the Frolicher-Nijenhuis bracket (see Theorem 5.1) and apply this fact to double-linear connections $\Gamma: K \rightarrow T^{*} M \otimes T K$ in $K$ (see Theorem 6.3).

By the local description of double vector bundles, presented in 8 , the notion of double vector bundles in the sense of the present paper is equivalent to the one in the book [11. Product preserving (gauge) bundle functors are studied in [1, 6, 7 , 9, 10, 12, 13, 14, 16, 17, 18. Liftings of vector fields to product preserving (gauge) bundle functors are studied in [5, 10, 14]. Complete lifting of general connections on fibered manifolds to Weil functors is studied in 7]. Complete lifting of semi-basic tangent valued $p$-forms on fibered manifolds to Weil functors is studied in [2, 3]. Complete lifting of linear semi-basic tangent valued forms to product preserving gauge bundle functors on vector bundles is studied in [15]. The Frolicher-Nijenhuis bracket on projectable tangent valued forms is studied in [4].

\section{Preliminaries}

Let $K$ be a double vector bundle. Let $M$ be the basis of $K$ and $\pi: K \rightarrow M$ be the projection.

Lemma 2.1. Let $Z, Z_{1}$ be double-linear vector fields on $K, \alpha$ a real number and $f: M \rightarrow \mathbf{R}$ a map. Then $Z+Z_{1}, \alpha Z, f \circ \pi \cdot Z$ and $\left[Z, Z_{1}\right]$ are double linear vector fields on $K$.

Proof. Using $\mathcal{D} \mathcal{V B}$-charts, we may assume $K=\mathbf{R}^{m_{1}, m_{2}, n_{1}, n_{2}}$. Let $x^{1}, \ldots, x^{m_{1}}$, $u^{1}, \ldots, u^{m_{2}}, v^{1}, \ldots, v^{n_{1}}, w^{1}, \ldots, w^{n_{2}}$ be the usual coordinates. A map $f: K \rightarrow K$ 
is a $\mathcal{D} \mathcal{V B}$-map if and only if it is of the form

$$
\begin{aligned}
x^{i} \circ f & =\alpha^{i}(x), \quad i=1, \ldots, m_{1}, \\
u^{j} \circ f & =\sum_{j_{1}=1}^{m_{2}} \beta_{j_{1}}^{j}(x) u^{j_{1}}, \quad j=1, \ldots, m_{2}, \\
v^{k} \circ f & =\sum_{k_{1}=1}^{n_{1}} \gamma_{k_{1}}^{k}(x) v^{k_{1}}, \quad k=1, \ldots, n_{1}, \\
w^{l} \circ f & =\sum_{l_{1}=1}^{n_{2}} \gamma_{l_{1}}^{l}(x) w^{l_{1}}+\sum_{j_{1}=1}^{m_{2}} \sum_{k_{1}=1}^{n_{1}} \sigma_{j_{1} k_{1}}^{l}(x) u^{j_{1}} v^{k_{1}}, \quad l=1, \ldots, n_{2},
\end{aligned}
$$

where $x=\left(x^{1}, \ldots, x^{m_{1}}\right)$. Consequently, a vector field $Z$ on $K$ is double linear if and only if it is of the form

$$
\begin{aligned}
Z= & \sum_{i=1}^{m_{1}} a^{i}(x) \frac{\partial}{\partial x^{i}}+\sum_{j, j^{1}=1}^{m_{2}} b_{j}^{j_{1}}(x) u^{j} \frac{\partial}{\partial u^{j_{1}}}+\sum_{k, k_{1}=1}^{n_{1}} c_{k}^{k_{1}}(x) v^{k} \frac{\partial}{\partial v^{k_{1}}} \\
& +\sum_{l, l^{1}=1}^{n_{2}} e_{l}^{l_{1}}(x) w^{l} \frac{\partial}{\partial w^{l_{1}}}+\sum_{j_{2}=1}^{m_{2}} \sum_{k_{2}=1}^{n_{1}} \sum_{l_{2}=1}^{n_{2}} f_{j_{2} k_{2}}^{l_{2}}(x) u^{j_{2}} v^{k_{2}} \frac{\partial}{\partial w^{l_{2}}} .
\end{aligned}
$$

The lemma is now clear.

Now, we treat $K$ as a fibered manifold over $M$ or (generally) let $\pi: K \rightarrow M$ be an arbitrary fibered manifold.

Definition 2.2. A projectable semi-basic tangent valued $p$-form on $K$ is a section $\varphi: K \rightarrow \wedge^{p} T^{*} M \otimes T K$ such that $\varphi\left(X_{1}, \ldots, X_{p}\right)$ is a projectable vector field on $K$.

Given a projectable semi-basic tangent valued $p$-form $\varphi: K \rightarrow \wedge^{p} T^{*} M \otimes T K$ we have the underlying tangent valued $p$-form $\varphi: M \rightarrow \wedge^{p} T^{*} M \otimes T M$ on $M$ such that $\varphi\left(X_{1}, \ldots, X_{p}\right)$ is the underlying vector field of the projectable vector field $\varphi\left(X_{1}, \ldots, X_{p}\right)$ for any vector fields $X_{1}, \ldots, X_{p}$ on $M$.

The following fact is well known; see e.g. [3, 4.

Lemma 2.3. Given a projectable semi-basic tangent-valued p-form $\varphi: K \rightarrow$ $\wedge^{p} T^{*} M \otimes T K$ on $K$ and a projectable semi-basic tangent valued $q$-form $\psi: K \rightarrow$ $\wedge^{q} T^{*} M \otimes T K$ on $K$ there exists a (unique) projectable semi-basic tangent valued 
$(p+q)$-form $[[\varphi, \psi]]: K \rightarrow \wedge^{p+q} T^{*} M \otimes T K$ on $K$ such that

$[[\varphi, \psi]]\left(X_{1}, \ldots, X_{p+q}\right)$

$$
\begin{aligned}
= & \frac{1}{p ! q !} \sum_{\sigma} \operatorname{sgn} \sigma \cdot\left[\varphi\left(X_{\sigma 1}, \ldots, X_{\sigma p}\right), \psi\left(X_{\sigma(p+1)}, \ldots, X_{\sigma(p+q)}\right)\right] \\
& +\frac{-1}{p !(q-1) !} \sum_{\sigma} \operatorname{sgn} \sigma \cdot \psi\left(\left[\underline{\varphi}\left(X_{\sigma 1}, \ldots, X_{\sigma p}\right), X_{\sigma(p+1)}\right], X_{\sigma(p+2)}, \ldots\right) \\
& +\frac{(-1)^{p q}}{(p-1) ! q !} \sum_{\sigma} \operatorname{sgn} \sigma \cdot \varphi\left(\left[\underline{\psi}\left(X_{\sigma 1}, \ldots, X_{\sigma q}\right), X_{\sigma(q+1)}\right], X_{\sigma(q+2)}, \ldots\right) \\
& +\frac{(-1)^{p-1}}{(p-1) !(q-1) ! 2 !} \sum_{\sigma} \operatorname{sgn} \sigma \cdot \psi\left(\underline{\varphi}\left(\left[X_{\sigma 1}, X_{\sigma 2}\right], X_{\sigma 3}, \ldots\right), X_{\sigma(p+2)}, \ldots\right) \\
& +\frac{(-1)^{(p-1) q}}{(p-1) !(q-1) ! 2 !} \sum_{\sigma} \operatorname{sgn} \sigma \cdot \varphi\left(\underline{\psi}\left(\left[X_{\sigma_{1}}, X_{\sigma 2}\right], X_{\sigma 3}, \ldots\right), X_{\sigma(q+2)}, \ldots\right)
\end{aligned}
$$

for any vector fields $X_{1}, \ldots, X_{p+q}$ on $M$, where sums are over all permutations $\sigma:\{1, \ldots, p+q\} \rightarrow\{1, \ldots, p+q\}$ and $\operatorname{sgn} \sigma$ is the signum of $\sigma$.

The underlying tangent valued $(p+q)$-form of $[[\varphi, \psi]]$ is $[[\underline{\varphi}, \underline{\psi}]]$.

Definition 2.4. The bracket $[[-,-]]$ is called the Frolicher-Nijenhuis bracket.

Proposition 2.5. Let $K$ be a double vector bundle with basis $M$. Let $\varphi: K \rightarrow$ $\wedge^{p} T^{*} M \otimes T K$ be a double-linear (then projectable) semi-basic tangent valued p-form on $K$ and let $\psi: K \rightarrow \wedge^{q} T^{*} M \otimes T K$ be a double-linear semi-basic tangent valued $q$-form on $K$. Then the Frolicher-Nijenhuis bracket $[[\varphi, \psi]]: K \rightarrow \wedge^{p+q} T^{*} M \otimes T K$ is a double-linear semi-basic tangent valued $(p+q)$-form on $K$.

Proof. It follows from formula (2.2), Lemma 2.1 and Definition 1.4

We end this section with the $\mathcal{D} \mathcal{V B}$-version of the well-known fact of the simplicity of vector fields.

Lemma 2.6. Let $Z$ be a double linear vector field on a double vector bundle $K$ such that the underlying vector field $\underline{Z}$ on basis $M$ is nonzero at a point $x_{o} \in M$. Then there exists a local $\mathcal{D} \mathcal{V B}$-coordinate system $\left(x^{1}, \ldots\right)$ on $K$ with centrum $x_{o}$ such that $Z=\frac{\partial}{\partial x^{1}}$.

Proof. The proof is quite similar to that of the manifold case. We may assume that $K=\mathbf{R}^{m_{1}, m_{2}, n_{1}, n_{2}}, x_{o}=0$ and $\underline{Z}_{\mid 0}=\frac{\partial}{\partial x^{1}} \mid 0$. Let $\left\{\varphi_{t}\right\}$ be the flow of $Z$. Then $\Phi: K \rightarrow K$ given by $\Phi\left(x^{1}, \ldots\right)=\varphi_{x_{1}}\left(0, x^{2}, \ldots\right)$ is a local $\mathcal{D} \mathcal{V B}$-isomorphism sending $\frac{\partial}{\partial x^{1}}$ to $Z$.

\section{ON THE COMPLETE LIFTING OF DOUBLE-LINEAR VECTOR FIELDS TO PPGB-FUNCTORS ON DOUBLE VECTOR BUNDLES}

Let $F: \mathcal{D V} \mathcal{B} \rightarrow \mathcal{F M}$ be a ppgb-functor. We know that $F: \mathcal{D V} \mathcal{B} \rightarrow \mathcal{D V} \mathcal{B}$. Let $Z$ be a double-linear vector field on a double vector bundle $K$. 
Definition 3.1. The complete lift of $Z$ to $F$ is the double-linear vector field $\mathcal{F} Z$ on $F K$ corresponding to the flow $\left\{F \varphi_{t}\right\}$, where $\left\{\varphi_{t}\right\}$ is the flow of $Z$.

Lemma 3.2. If $\varphi: K \rightarrow K_{1}$ is a (locally defined) $\mathcal{D} \mathcal{V B}$-isomorphism, then $\mathcal{F}\left(\varphi_{*} Z\right)=(F \varphi)_{*} \mathcal{F} Z$.

Proof. The flow of $\varphi_{*} Z$ is $\left\{\varphi \circ \varphi_{t} \circ \varphi^{-1}\right\}$. Then the flow of $\mathcal{F}\left(\varphi_{*} Z\right)$ is $\left\{F \varphi \circ F \varphi_{t} \circ\right.$ $\left.(F \varphi)^{-1}\right\}$. The last flow is the one of $(F \varphi)_{*} \mathcal{F} Z$.

Lemma 3.3. If $\alpha$ is a real number, then $\mathcal{F}(\alpha Z)=\alpha \mathcal{F} Z$. Consequently, $\mathcal{F}(\alpha Z+$ $\left.\alpha_{1} Z_{1}\right)=\alpha F Z+\alpha_{1} \mathcal{F} Z_{1}$ for any real numbers $\alpha$ and $\alpha_{1}$ and any double linear vector fields $Z$ and $Z_{1}$ on $K$.

Proof. If $\left\{\varphi_{t}\right\}$ is the flow of $Z$, then $\left\{\varphi_{\alpha t}\right\}$ is the flow of $\alpha Z$. So, $\left\{F \varphi_{\alpha t}\right\}$ is the flow of $\mathcal{F}(\alpha Z)$ and of $\alpha \mathcal{F} Z$. Hence, $\mathcal{F}$ is $\mathbf{R}$-linear because of the homogeneous function theorem and the nonlinear Peetre theorem [7].

Let $\diamond^{F}: U^{F} \times V^{F} \rightarrow W^{F}$ be the $A^{F}$-bilinear map corresponding to $F$.

Lemma 3.4. Let $Z$ be a double linear vector field on a double vector bundle $K$ with basis $M$ and let $a \in A^{F}$. Then $\operatorname{af}(a) \circ \mathcal{F} Z$ is a double linear vector field on $F K$.

Proof. We may assume that the underlying vector field $\underline{Z}$ is nowhere vanishing. Then using $\mathcal{D} \mathcal{V B}$-charts and Lemma 2.6 we may assume that $Z=\frac{\partial}{\partial x^{1}}$ and $K=$ $\mathbf{R}^{m_{1}, m_{2}, n_{1}, n_{2}}$. Then $F K=\left(A^{F}\right)^{m_{1}} \times\left(U^{F}\right)^{m_{2}} \times\left(V^{F}\right)^{n_{1}} \times\left(W^{F}\right)^{n_{2}}$ and af $(a) \circ \mathcal{F} Z$ can be treated as a vector field on $\left(A^{F}\right)^{m_{1}}$ (and consequently as a double linear vector field on $F K$ ).

By Lemma 2.1, if $Z$ and $Z_{1}$ are double linear vector fields on $K$ then so is [ $\left.Z, Z_{1}\right]$.

Proposition 3.5. For any double linear vector fields $Z$ and $Z_{1}$ on $K$ and any $a, a_{1} \in A^{F}$ we have

$$
\left[\operatorname{af}(a) \circ \mathcal{F} Z, \operatorname{af}\left(a_{1}\right) \circ \mathcal{F} Z_{1}\right]=\operatorname{af}\left(a a_{1}\right) \circ \mathcal{F}\left(\left[Z, Z_{1}\right]\right) .
$$

Proof. We may assume that $K=\mathbf{R}^{m_{1}, m_{2}, n_{1}, n_{2}}, Z=\frac{\partial}{\partial x^{1}}$ and $Z_{1}=f\left(x^{1}, \ldots, x^{m_{1}}\right) Z_{2}$, where $Z_{2} \in\left\{\frac{\partial}{\partial x^{2}}, u^{j} \frac{\partial}{\partial u^{j_{1}}}, v^{k} \frac{\partial}{\partial v^{k_{1}}}, w^{l} \frac{\partial}{\partial w^{l_{1}}}, u^{j} v^{k} \frac{\partial}{\partial w^{l}}\right\}$.

If $Z_{2}=\frac{\partial}{\partial x^{i}}$, then the formula is the well-know one for usual Weil functors on manifolds. For other values of $Z_{2}$, using formula (3.2) (below) and the known formula $a \mathcal{F} Z\left(a_{1} F f\right)=a a_{1} F(Z(f))$ for usual Weil functors on manifolds, we get $\left[\operatorname{af}(a) \circ \mathcal{F} Z, \operatorname{af}\left(a_{1}\right) \circ \mathcal{F}\left(f Z_{2}\right)\right]=\left[a \cdot \mathcal{F} Z, a_{1} F f \cdot \mathcal{F} Z_{2}\right]=a \mathcal{F} Z\left(a_{1} F f\right) \cdot \mathcal{F} Z_{2}=$ $a a_{1} F(Z(f)) \cdot \mathcal{F} Z_{2}=a a_{1} \cdot \mathcal{F}\left(Z(f) Z_{2}\right)=\operatorname{af}\left(a a_{1}\right) \circ \mathcal{F}\left(\left[Z, Z_{1}\right]\right)$.

Lemma 3.6. Let $Z$ be a double linear vector field on $K$ and let $f: M \rightarrow \mathbf{R}$ be a map. Then

$$
\mathcal{F}(f \circ \pi \cdot Z)=F f \circ F \pi \cdot \mathcal{F} Z,
$$

where $\pi: K \rightarrow M$ is the projection (we treat $M$ as a $\mathcal{D} \mathcal{V B}$-object and $\pi$ as a $\mathcal{D V B}$-map in the obvious way) and $F f: F M \rightarrow F \mathbf{R}=A^{F}$. Here (in the right of the formula) $a \cdot y:=\operatorname{af}(a)(y)$ for $a \in A^{F}$ and $y \in T F K$. 
Proof. By Lemma 2.1 $f \circ \pi \cdot Z$ is double linear. So, both sides of 3.2 make sense. By the linearity of $\mathcal{F}$, we may assume that $Z$ is not $\pi$-vertical. Then by Lemma 2.6 we may assume that $K=\mathbf{R}^{m_{1}, m_{2}, n_{1}, n_{2}}$ and $Z=\frac{\partial}{\partial x^{1}}$. Then we may additional assume that $K=M$ is a manifold, $Z$ is a vector field on $M$ and $F$ is a Weil functor on manifolds. Then our lemma is the (well known for Weil functors on manifolds) formula $\mathcal{F}(f Z)=F f \cdot \mathcal{F} Z$.

\section{On the COMPlete Lifting of DOUble-Linear SEMi-BASiC TANGEnT VALUED $p$-FORMS TO PPGB-FUNCTORS ON DOUBLE VECTOR BUNDLES}

For a moment, let $F$ be a ppgb-functor (Weil functor) on manifolds. Let $\omega \in$ $\Omega^{p}(M)$ be a $p$-form on a manifold $M$. Then $\omega: T M \times_{M} \ldots \times_{M} T M \rightarrow \mathbf{R}$ is a fiber skew $p$-linear map. Applying $F$, we get the fibre skew $p$-linear (over $A^{F}$ ) map $F \omega: F T M \times_{F M} \ldots \times_{F M} F T M \rightarrow A^{F}$ (this is a well-known fact for Weil functors on manifolds). Then applying the exchange isomorphism $\eta_{M}: T F M \rightarrow F T M$, which is a vector bundle isomorphism (this is also a well-known fact for Weil functors on manifolds), we obtain the $A^{F}$-valued $p$-form

$$
\mathcal{F} \omega:=F \omega \circ\left(\eta_{M} \times \ldots \times \eta_{M}\right): T F M \times_{F M} \ldots \times_{F M} T F M \rightarrow A^{F}
$$

over $F M$.

Lemma 4.1. $\mathcal{F} \omega$ is the unique $A^{F}$-valued $p$-form on $F M$ such that

$$
\mathcal{F} \omega\left(\operatorname{af}\left(a_{1}\right) \circ \mathcal{F} X_{1}, \ldots, \operatorname{af}\left(a_{p}\right) \circ \mathcal{F} X_{p}\right)=a_{1} \cdot \ldots \cdot a_{p} \cdot F\left(\omega\left(X_{1}, \ldots, X_{p}\right)\right)
$$

for any vector fields $X_{1}, \ldots, X_{p}$ on $M$ and any $a_{1}, \ldots, a_{p} \in A^{F}$.

Proof. The uniqueness is a consequence of the well-known fact for Weil functors on manifolds that the vector fields af $(a) \circ \mathcal{F} X$ generate over $C^{\infty}(M)$ the vector space $\mathcal{X}(F M)$. Formula (4.1) follows from the well-known (for Weil functors on manifolds) equalities $\mathcal{F} X=\eta_{M}^{-1} \circ F X$ and $\eta_{M} \circ$ af $(a)=a \cdot \eta_{M}$.

Definition 4.2. The $A^{F}$-valued $p$-form on $F M$ satisfying 4.1 is called the complete lift of $\omega$ to $F$.

For the rest of this section, let $F: \mathcal{D V} \mathcal{B} \rightarrow \mathcal{F} \mathcal{M}$ be a ppgb-functor.

Let $x^{1}, \ldots, x^{m_{1}}, u^{1}, \ldots, u^{m_{2}}, v^{1}, \ldots, v^{n_{1}}, w^{1}, \ldots, w^{n_{2}}$ be the usual coordinates on $\mathbf{R}^{m_{1}, m_{2}, n_{1}, n_{2}}$.

Because of the local expression (2.1) of double-linear vector fields and of the Definition 1.4 of double-linear semi-basic tangent valued $p$-forms, any double-linear semi-basic tangent valued $p$-form $\varphi$ on $\mathbf{R}^{m_{1}, m_{2}, n_{1}, n_{2}}$ is of the form

$$
\begin{aligned}
\varphi= & \sum_{i=1}^{m_{1}} \varphi^{i} \otimes_{\mathbf{R}} \frac{\partial}{\partial x^{i}}+\sum_{j, j_{1}=1}^{m_{2}} \psi_{j_{1}}^{j} \otimes_{\mathbf{R}} u^{j_{1}} \frac{\partial}{\partial u^{j}} \\
& +\sum_{k, k_{1}=1}^{n_{1}} \chi_{k_{1}}^{k} \otimes_{\mathbf{R}} v^{k_{1}} \frac{\partial}{\partial v^{k}}+\sum_{l, l_{1}=1}^{n_{2}} \xi_{l_{1}}^{l} \otimes_{\mathbf{R}} w^{l_{1}} \frac{\partial}{\partial w^{l}} \\
& +\sum_{j=1}^{m_{2}} \sum_{k=1}^{n_{1}} \sum_{l=1}^{n_{2}} \rho_{j k}^{l} \otimes_{\mathbf{R}} u^{j} v^{k} \frac{\partial}{\partial w^{l}}
\end{aligned}
$$


for unique $p$-forms $\varphi^{i}, \psi_{j_{1}}^{j}, \chi_{k_{1}}^{k}, \xi_{l_{1}}^{l}, \rho_{j k}^{l}$ on $\mathbf{R}^{m}$, where $\left(\omega \otimes_{\mathbf{R}} Z\right)\left(X_{1}, \ldots, X_{p}\right):=$ $\omega\left(X_{1}, \ldots, X_{p}\right) \circ \pi \cdot Z$.

For any such $\varphi$ we define its complete lift $\mathcal{F} \varphi$ by

$$
\begin{aligned}
\mathcal{F} \varphi:= & \sum_{i=1}^{m_{1}} \mathcal{F} \varphi^{i} \otimes_{A^{F}} \mathcal{F} \frac{\partial}{\partial x^{i}}+\sum_{j, j_{1}=1}^{m_{2}} \mathcal{F} \psi_{j_{1}}^{j} \otimes_{A^{F}} \mathcal{F}\left(u^{j_{1}} \frac{\partial}{\partial u^{j}}\right) \\
& +\sum_{k, k_{1}=1}^{n_{1}} \mathcal{F} \chi_{k_{1}}^{k} \otimes_{A^{F}} \mathcal{F}\left(v^{k_{1}} \frac{\partial}{\partial v^{k}}\right)+\sum_{l, l_{1}=1}^{n_{2}} \mathcal{F} \xi_{l_{1}}^{l} \otimes_{A^{F}} \mathcal{F}\left(w^{l_{1}} \frac{\partial}{\partial w^{l}}\right) \\
& +\sum_{j=1}^{m_{2}} \sum_{k=1}^{n_{1}} \sum_{l=1}^{n_{2}} \mathcal{F} \rho_{j k}^{l} \otimes_{A^{F}} \mathcal{F}\left(u^{j} v^{k} \frac{\partial}{\partial w^{l}}\right)
\end{aligned}
$$

where $\left(\mathcal{F} \omega \otimes_{A^{F}} \mathcal{F} Z\right)\left(Y_{1}, \ldots, Y_{p}\right):=\mathcal{F} \omega\left(Y_{1}, \ldots, Y_{p}\right) \circ F \pi \cdot \mathcal{F} Z$ for $Y_{1}, \ldots, Y_{p} \in$ $\mathcal{X}\left(F \mathbf{R}^{m_{1}}\right)$.

Proposition 4.3. The complete lift $\mathcal{F} \varphi$ as in 4.2 is the unique double-linear semi-basic tangent valued $p$-form on $F \mathbf{R}^{m_{1}, m_{2}, n_{1}, n_{2}}$ such that

$$
\mathcal{F} \varphi\left(\operatorname{af}\left(a_{1}\right) \circ \mathcal{F} X_{1}, \ldots, \operatorname{af}\left(a_{p}\right) \circ \mathcal{F} X_{p}\right)=\operatorname{af}\left(a_{1} \cdot \ldots \cdot a_{p}\right) \circ \mathcal{F}\left(\varphi\left(X_{1}, \ldots, X_{p}\right)\right)
$$

for any $a_{1}, \ldots, a_{p} \in A^{F}$ and any $X_{1}, \ldots, X_{p} \in \mathcal{X}\left(\mathbf{R}^{m_{1}}\right)$.

Proof. The uniqueness is clear because the vector fields af $(a) \circ \mathcal{F} X$ for $a \in A^{F}$ and $X \in \mathcal{X}\left(\mathbf{R}^{m_{1}}\right)$ generate (over $C^{\infty}\left(F \mathbf{R}^{m_{1}}\right)$ ) the vector space $\mathcal{X}\left(F \mathbf{R}^{m_{1}}\right)$. This is a well-known fact for Weil functors on manifolds.

Now, we prove (4.3). Since both sides of (4.3) are linear in $\varphi$, we may assume that $\varphi=\omega \otimes_{\mathbf{R}} Z$, where $\omega \in \Omega^{p}\left(\mathbf{R}^{m_{1}}\right)$ and $Z \in\left\{\frac{\partial}{\partial x^{i}}, u^{j_{1}} \frac{\partial}{\partial u^{j}}, v^{k_{1}} \frac{\partial}{\partial v^{k}}, w^{l_{1}} \frac{\partial}{\partial w^{l}}, u^{j} v^{k} \frac{\partial}{\partial w^{l}}\right\}$. Then by (4.2), 4.1) and (3.2) we have

$$
\begin{aligned}
\mathcal{F} \varphi\left(\operatorname{af}\left(a_{1}\right) \circ \mathcal{F} X_{1}, \ldots, \operatorname{af}\left(a_{p}\right) \circ \mathcal{F} X_{p}\right) \\
\quad=\mathcal{F}\left(\omega \otimes_{\mathbf{R}} Z\right)\left(\operatorname{af}\left(a_{1}\right) \circ \mathcal{F} X_{1}, \ldots, \operatorname{af}\left(a_{p}\right) \circ \mathcal{F} X_{p}\right) \\
\quad=\left(\mathcal{F} \omega \otimes_{A^{F}} \mathcal{F} Z\right)\left(\operatorname{af}\left(a_{1}\right) \circ \mathcal{F} X_{1}, \ldots, \operatorname{af}\left(a_{p}\right) \circ \mathcal{F} X_{p}\right) \\
\quad=\mathcal{F} \omega\left(\operatorname{af}\left(a_{1}\right) \circ \mathcal{F} X_{1}, \ldots, \operatorname{af}\left(a_{p}\right) \circ \mathcal{F} X_{p}\right) \circ F \pi \cdot \mathcal{F} Z \\
\quad=a_{1} \cdot \ldots \cdot a_{p} \cdot F\left(\omega\left(X_{1}, \ldots, X_{p}\right)\right) \circ F \pi \cdot \mathcal{F} Z \\
\quad=a_{1} \cdot \ldots \cdot a_{p} \cdot \mathcal{F}\left(\omega\left(X_{1}, \ldots, X_{p}\right) \circ \pi \cdot Z\right) \\
\quad=a_{1} \cdot \ldots \cdot a_{p} \cdot \mathcal{F}\left(\left(\omega \otimes_{\mathbf{R}} Z\right)\left(X_{1}, \ldots, X_{p}\right)\right) \\
\quad=\operatorname{af}\left(a_{1} \cdot \ldots \cdot a_{p}\right) \circ \mathcal{F}\left(\varphi\left(X_{1}, \ldots, X_{p}\right)\right)
\end{aligned}
$$

Lemma 4.4. For any (local) double vector bundle isomorphism $f: \mathbf{R}^{m_{1}, m_{2}, n_{1}, n_{2}} \rightarrow$ $\mathbf{R}^{m_{1}, m_{2}, n_{1}, n_{2}}$ and any double-linear semi-basic tangent valued $p$-form $\varphi$ on the double vector bundle $\mathbf{R}^{m_{1}, m_{2}, n_{1}, n_{2}}$, we have $(F f)_{*} \mathcal{F} \varphi=\mathcal{F}\left(f_{*} \varphi\right)$. 
Proof. We have

$$
\begin{array}{rl}
(F f)_{*} & \mathcal{F} \varphi\left(\operatorname{af}\left(a_{1}\right) \circ \mathcal{F} X_{1}, \ldots, \operatorname{af}\left(a_{p}\right) \mathcal{F} X_{p}\right) \\
& =\mathcal{F} \varphi\left(F f_{*}^{-1}\left(\operatorname{af}\left(a_{1}\right) \circ \mathcal{F} X_{1}\right), \ldots, F f_{*}^{-1}\left(\operatorname{af}\left(a_{p}\right) \circ \mathcal{F} X_{p}\right)\right) \\
& =\mathcal{F} \varphi\left(\operatorname{af}\left(a_{1}\right) \circ \mathcal{F}\left(f_{*}^{-1} X_{1}\right), \ldots, \operatorname{af}\left(a_{p}\right) \circ \mathcal{F}\left(f_{*}^{-1} X_{p}\right)\right) \\
& =\operatorname{af}\left(a_{1} \cdot \ldots \cdot a_{p}\right) \circ \mathcal{F} \varphi\left(\mathcal{F}\left(f_{*}^{-1} X_{1}\right), \ldots, \mathcal{F}\left(f_{*}^{-1} X_{p}\right)\right) \\
& =\operatorname{af}\left(a_{1} \cdot \ldots \cdot a_{p}\right) \circ \mathcal{F}\left(\varphi\left(f_{*}^{-1} X_{1}, \ldots, f_{*}^{-1} X_{p}\right)\right) \\
& =\operatorname{af}\left(a_{1} \cdot \ldots \cdot a_{p}\right) \circ \mathcal{F}\left(\left(f_{*} \varphi\right)\left(X_{1}, \ldots, X_{p}\right)\right) \\
& =\mathcal{F}\left(f_{*} \varphi\right)\left(\operatorname{af}\left(a_{1}\right) \cdot \mathcal{F} X_{1}, \ldots, \operatorname{af}\left(a_{p}\right) \cdot \mathcal{F} X_{p}\right) .
\end{array}
$$

Now, applying the uniqueness case of Proposition 4.3 (or, better, the sentence of the proof of the uniqueness case of Proposition 4.3 we end the proof.

We are now in a position to prove the following result.

Theorem 4.5. Let $F$ be a ppgb-functor on $\mathcal{D} \mathcal{V B}$. Let $\varphi: K \rightarrow \wedge^{p} T^{*} M \otimes T K$ be a double-linear semi-basic tangent valued $p$-form on a double vector bundle $K$ with basis $M$. Then there exists one and only one double-linear semi-basic tangent valued p-form $\mathcal{F} \varphi: F K \rightarrow \wedge^{p} T^{*} F M \otimes T F K$ on $F K$ such that

$$
\mathcal{F} \varphi\left(\operatorname{af}\left(a_{1}\right) \circ \mathcal{F} X_{1}, \ldots, \text { af }\left(a_{p}\right) \circ \mathcal{F} X_{p}\right)=\operatorname{af}\left(a_{1} \cdot \ldots \cdot a_{p}\right) \circ \mathcal{F}\left(\varphi\left(X_{1}, \ldots, X_{p}\right)\right)
$$

for any vector fields $X_{1}, \ldots, X_{p}$ on $M$ and any $a_{1}, \ldots, a_{p} \in A^{F}$.

Proof. Using $\mathcal{D} \mathcal{V B}$-charts on $K$, we spread the complete lifting of double-linear semi-basic tangent valued $p$-forms on $\mathbf{R}^{m_{1}, m_{2}, n_{1}, n_{2}}$ to the one on $K$. This is possible because of Lemma 4.4 .

\section{The COMPlete Lifting OF DOUble-LineAR SEMI-BASiC TANGENT VAlued p-FORMS PRESERVES THE FROLICHER-NIJENHUIS BRACKET}

Let $F$ be a ppgb-functor on $\mathcal{D} \mathcal{V B}$. Then $F: \mathcal{D} \mathcal{V B} \rightarrow \mathcal{D} \mathcal{V B}$.

Let $\varphi: K \rightarrow \wedge^{p} T^{*} M \otimes T K$ be a double-linear semi-basic tangent valued $p$-form on $K$ and let $\psi: K \rightarrow \wedge^{q} T^{*} M \otimes T K$ be a double-linear semi-basic tangent valued $q$-form on $K$. We can lift $\varphi$ and $\psi$ to $F K$ and obtain a double-linear semi-basic tangent valued $p$-form $\mathcal{F} \varphi$ on $F K$ and a double-linear semi-basic tangent valued $q$-form $\mathcal{F} \psi$ on $F K$. Then we can produce the Frolicher-Nijenhuis bracket $[[\mathcal{F} \varphi, \mathcal{F} \psi]]$. By Proposition 2.5. this bracket is a double-linear semi-basic tangent valued $(p+q)$ form on $F K$.

On the other hand, by Proposition 2.5 the Frolicher-Nijenhuis bracket $[[\varphi, \psi]]$ is a double-linear semi-basic tangent valued $(p+q)$-form on $K$. So, we can lift it and obtain a double-linear semi-basic tangent valued $(p+q)$-form $\mathcal{F}([[\varphi, \psi]])$ on $F K$.

Theorem 5.1. We have

$$
\mathcal{F}([[\varphi, \psi]])=[[\mathcal{F} \varphi, \mathcal{F} \psi]]
$$


Proof. For any $a_{1}, \ldots, a_{p+1} \in A^{F}$ and vector fields $X_{1}, \ldots, X_{p+q}$ on $M$ we have

$$
\begin{aligned}
& {\left[\mathcal{F} \varphi\left(\operatorname{af}\left(a_{1}\right) \circ \mathcal{F} X_{1}, \ldots, \text { af }\left(a_{p}\right) \circ \mathcal{F} X_{p}\right),\right.} \\
& \left.\mathcal{F} \psi\left(\operatorname{af}\left(a_{p+1}\right) \circ \mathcal{F} X_{p+1}, \ldots, \text { af }\left(a_{p+q}\right) \circ \mathcal{F} X_{p+q}\right)\right] \\
& =\operatorname{af}\left(a_{1} \cdot \ldots \cdot a_{p+q}\right) \circ \mathcal{F}\left(\left[\varphi\left(X_{1}, \ldots, X_{p}\right), \psi\left(X_{p+1}, \ldots, X_{p+q}\right)\right]\right) \text {. }
\end{aligned}
$$

Indeed, applying formulas (4.4) and (3.1) we easily get

$$
\begin{aligned}
& {\left[\mathcal{F} \varphi\left(\operatorname{af}\left(a_{1}\right) \circ \mathcal{F} X_{1}, \ldots, \operatorname{af}\left(a_{p}\right) \circ \mathcal{F} X_{p}\right),\right.} \\
& \qquad \begin{array}{l}
\left.\mathcal{F} \psi\left(\operatorname{af}\left(a_{p+1}\right) \circ \mathcal{F} X_{p+1}, \ldots, \operatorname{af}\left(a_{p+q}\right) \circ \mathcal{F} X_{p+q}\right)\right] \\
=\left[\operatorname{af}\left(a_{1} \cdot \ldots \cdot a_{p}\right) \circ \mathcal{F}\left(\varphi\left(X_{1}, \ldots, X_{p}\right)\right),\right. \\
\left.\quad \operatorname{af}\left(a_{p+1} \cdot \ldots \cdot a_{p+q}\right) \circ \mathcal{F}\left(\psi\left(X_{p+1}, \ldots, X_{p+q}\right)\right)\right] \\
=\operatorname{af}\left(a_{1} \cdot \ldots \cdot a_{p+q}\right) \circ \mathcal{F}\left(\left[\varphi\left(X_{1}, \ldots, X_{p}\right), \psi\left(X_{p+1}, \ldots, X_{p+q}\right)\right]\right) .
\end{array}
\end{aligned}
$$

Similarly, we have

$$
\begin{aligned}
\mathcal{F} \psi\left(\left[\underline { \mathcal { F } \varphi } \left(\operatorname{af}\left(a_{1}\right)\right.\right.\right. & \left.\left.\circ \mathcal{F} X_{1}, \ldots, \operatorname{af}\left(a_{p}\right) \circ \mathcal{F} X_{p}\right), \operatorname{af}\left(a_{p+1}\right) \circ \mathcal{F} X_{p+1}\right] \\
\operatorname{af}\left(a_{p+2}\right) & \left.\circ \mathcal{F} X_{p+2}, \ldots, \operatorname{af}\left(a_{p+q}\right) \circ \mathcal{F} X_{p+q}\right) \\
\quad=\operatorname{af}\left(a_{1} \cdot \ldots \cdot a_{p+q}\right) & \circ \mathcal{F}\left(\psi\left(\left[\underline{\varphi}\left(X_{1}, \ldots, X_{p}\right), X_{p+1}\right], X_{p+2}, \ldots, X_{p+q}\right)\right)
\end{aligned}
$$

and

$$
\begin{aligned}
\mathcal{F} \psi\left(\underline{\mathcal{F} \varphi}\left(\left[\operatorname{af}\left(a_{1}\right) \circ \mathcal{F} X_{1}, \operatorname{af}\left(a_{2}\right) \circ \mathcal{F} X_{2}\right], \operatorname{af}\left(a_{3}\right) \circ \mathcal{F} X_{3}, \ldots, \text { af }\left(a_{p+1}\right) \circ \mathcal{F} X_{p+1}\right),\right. \\
\left.\operatorname{af}\left(a_{p+2}\right) \circ \mathcal{F} X_{p+2}, \ldots, \operatorname{af}\left(a_{p+q}\right) \circ \mathcal{F} X_{p+q}\right) \\
=\operatorname{af}\left(a_{1} \cdot \ldots \cdot a_{p+q}\right) \circ \mathcal{F}\left(\psi\left(\underline{\varphi}\left(\left[X_{1}, X_{2}\right], X_{3}, \ldots, X_{p+1}\right), X_{p+2}, \ldots, X_{p+q}\right)\right),
\end{aligned}
$$

and the same formulas with $\varphi$ replaced by $\psi$ and vice versa, and the same formulas with indices $1, \ldots, p+q$ replaced by $\sigma(1), \ldots, \sigma(p+q)$. Now, using the above formulas and formula 4.4 for $[[\varphi, \psi]]$ instead of $\varphi$ and formula $(2.2)$ on the Frolicher-Nijenhuis bracket $[[\varphi, \psi]]$ and formula $[2.2$ with $\varphi$ and $\psi$ replaced by $\mathcal{F} \varphi$ and $\mathcal{F} \psi$, and the $\mathbf{R}$-linearity of the complete lifting of vector fields (Lemma 3.3), 
we get

$$
\begin{aligned}
& \mathcal{F}([[\varphi, \psi]])\left(\operatorname{af}\left(a_{1}\right) \circ \mathcal{F} X_{1}, \ldots, \operatorname{af}\left(a_{p+q}\right) \circ \mathcal{F} X_{p+q}\right) \\
&=\operatorname{af}(a) \circ \mathcal{F}\left([[\varphi, \psi]]\left(X_{1}, \ldots, X_{p+q}\right)\right) \\
&=\frac{1}{p ! q !} \sum_{\sigma} \operatorname{sgn} \sigma \cdot \operatorname{af}(a) \circ \mathcal{F}\left(\left[\varphi\left(X_{\sigma 1}, \ldots, X_{\sigma p}\right), \psi\left(X_{\sigma(p+1)}, \ldots, X_{\sigma(p+q)}\right)\right]\right) \\
&+\frac{-1}{p !(q-1) !} \sum_{\sigma} \operatorname{sgn} \sigma \cdot \operatorname{af}(a) \circ \mathcal{F}\left(\psi\left(\left[\underline{\varphi}\left(X_{\sigma 1}, \ldots, X_{\sigma p}\right), X_{\sigma(p+1)}\right], X_{\sigma(p+2)}, \ldots\right)\right) \\
&+\frac{(-1)^{p q}}{(p-1) ! q !} \sum_{\sigma} \operatorname{sgn} \sigma \cdot \operatorname{af}(a) \circ \mathcal{F}\left(\varphi\left(\left[\underline{\psi}\left(X_{\sigma 1}, \ldots, X_{\sigma q}\right), X_{\sigma(q+1)}\right], X_{\sigma(q+2)}, \ldots\right)\right) \\
&+\frac{(-1)^{p-1}}{(p-1) !(q-1) ! 2 !} \sum_{\sigma} \operatorname{sgn} \sigma \cdot \operatorname{af}(a) \circ \mathcal{F}\left(\psi\left(\underline{\varphi}\left(\left[X_{\sigma 1}, X_{\sigma 2}\right], X_{\sigma 3}, \ldots\right), X_{\sigma(p+2)}, \ldots\right)\right) \\
&+\frac{(-1)^{(p-1) q}}{(p-1) !(q-1) ! 2 !} \sum_{\sigma} \operatorname{sgn} \sigma \cdot \operatorname{af}(a) \circ \mathcal{F}\left(\varphi\left(\underline{\psi}\left(\left[X_{\sigma_{1}}, X_{\sigma 2}\right], X_{\sigma 3}, \ldots\right), X_{\sigma(q+2)}, \ldots\right)\right) \\
&=\frac{1}{p ! q !} \sum_{\sigma} \operatorname{sgn} \sigma \cdot\left[\mathcal{F} \varphi\left(\operatorname{af}\left(a_{\sigma 1}\right) \circ \mathcal{F} X_{\sigma 1}, \ldots\right), \mathcal{F} \psi\left(\operatorname{af}\left(a_{\sigma(p+1)}\right) \circ \mathcal{F} X_{\sigma(p+1)}, \ldots\right)\right] \\
&+\frac{-1}{p !(q-1) !} \sum_{\sigma} \operatorname{sgn} \sigma \cdot \mathcal{F} \psi\left(\left[\underline{\mathcal{F} \varphi}\left(\operatorname{af}\left(a_{\sigma 1}\right) \circ \mathcal{F} X_{\sigma 1}, \ldots\right), \operatorname{af}\left(a_{\sigma(p+1)}\right) \circ \mathcal{F} X_{\sigma(p+1)}\right], \ldots\right) \\
&+\frac{(-1)^{p q}}{(p-1) ! q !} \sum_{\sigma} \operatorname{sgn} \sigma \cdot \mathcal{F} \varphi\left(\left[\underline{\mathcal{F} \psi}\left(\operatorname{af}\left(a_{\sigma 1}\right) \circ \mathcal{F} X_{\sigma 1}, \ldots\right), \operatorname{af}\left(a_{\sigma(p+1)}\right) \circ \mathcal{F} X_{\sigma(p+1)}\right], \ldots\right) \\
&+\frac{(-1)^{p-1}}{(p-1) !(q-1) ! 2 !} \sum_{\sigma} \operatorname{sgn} \sigma \cdot \mathcal{F} \psi\left(\mathcal{F} \varphi\left(\left[\operatorname{af}\left(a_{\sigma 1}\right) \circ \mathcal{F} X_{\sigma 1}, \operatorname{af}\left(a_{\sigma 2}\right) \circ \mathcal{F} X_{\sigma 2}\right], \ldots\right), \ldots\right) \\
&+\frac{(-1)^{(p-1) q}}{(p-1) !(q-1) ! 2 !} \sum_{\sigma} \operatorname{sgn} \sigma \cdot \mathcal{F} \varphi\left(\mathcal{F} \psi\left(\left[\operatorname{af}\left(a_{\sigma 1}\right) \circ \mathcal{F} X_{\sigma 1}, \operatorname{af}\left(a_{\sigma 2}\right) \circ \mathcal{F} X_{\sigma 2}\right], \ldots\right), \ldots\right) \\
&= {[\mathcal{F} \varphi, \mathcal{F} \psi]]\left(\operatorname{af}\left(a_{1}\right) \circ \mathcal{F} X_{1}, \ldots, \operatorname{af}\left(a_{p+q}\right) \circ \mathcal{F} X_{p+q}\right), }
\end{aligned}
$$

for any vector fields $X_{1}, \ldots, X_{p+q}$ on $M$ and any $a_{1}, \ldots, a_{p+q} \in A^{F}$, where $a:=$ $a_{1} \cdot \ldots \cdot a_{p+q}$. Then, since the vector fields af $(a) \circ \mathcal{F} X$ generate (over $C^{\infty}(F M)$ ) the space $\mathcal{X}(F M)$, formula (5.1) holds.

\section{An APPlication to DOUble-Linear GENERAL CONNECTIONS}

Let $F$ be a ppgb-functor on $\mathcal{D V B}$.

In Definition 1.5 , we introduced the concept of double-linear connections $\Gamma$ in a double vector bundle $K$.

Lemma 6.1. Given a double linear connection $\Gamma$ in $K$, its complete lift $\mathcal{F} \Gamma$ is a double-linear connection in FK.

Proof. Since $\Gamma(X)$ is a double-linear vector field on $K$ with the underlying vector field equal to $X$, we have that $\mathcal{F} \Gamma(\operatorname{af}(a) \circ \mathcal{F} X)=\operatorname{af}(a) \cdot \mathcal{F}(\Gamma(X))$ is a double-linear vector field with the underlying vector field equal to af $(a) \circ \mathcal{F} X$. Consequently, for any vector field $Y \in \mathcal{X}(F M), \mathcal{F} \Gamma(Y)$ is a double linear vector field with the underlying vector field equal to $Y$. 
Definition 6.2. A curvature of a double linear connection $\Gamma$ in a double vector bundle $K$ is $\mathcal{R}_{\Gamma}:=\frac{1}{2}[[\Gamma, \Gamma]]: K \rightarrow \wedge^{2} T^{*} M \otimes V K$ (i.e., $\mathcal{R}_{\Gamma}(X, Y)=[\Gamma(X), \Gamma(Y)]-$ $\Gamma([X, Y]))$.

Theorem 6.3. We have

$$
\mathcal{R}_{\mathcal{F} \Gamma}=\mathcal{F}\left(\mathcal{R}_{\Gamma}\right) .
$$

Proof. It is clear because of $\mathcal{F}([[\Gamma, \Gamma]])=[[\mathcal{F} \Gamma, \mathcal{F} \Gamma]]$.

\section{ACKNOWLEDGEMENT}

I would like to thank the reviewer for his/her suggestion on notions.

\section{REFERENCES}

[1] G. N. Bushueva, Weil functors and product-preserving functors on the category of parameterdependent manifolds. Russian Math. (Iz. VUZ) 49 (2005), no. 5, 11-18. MR 2186866

[2] A. Cabras and I. Kolářr, Prolongation of tangent valued forms to Weil bundles, Arch. Math. (Brno) 31 (1995), no. 2, 139-145. MR 1357981

[3] A. Cabras and I. Kolář, Flow prolongation of some tangent valued forms, Czechoslovak Math. J. 58(133) (2008), no. 2, 493-504. MR 2411105

[4] J. Janyška, Natural operations with projectable tangent valued forms on a fibred manifold, Ann. Mat. Pura Appl. (4) 159 (1991), 171-187. MR 1145096

[5] I. Kolár, On the natural operators on vector fields, Ann. Global Anal. Geom. 6 (1988), no. 2, 109-117. MR 0982760

[6] I. Kolár, Weil bundles as generalized jet spaces, in Handbook of Global Analysis, 625-664, Elsevier Sci. B. V., Amsterdam, 2008. MR 2389643

[7] I. Kolář, P. W. Michor and J. Slovák, Natural Operations in Differential Geometry, SpringerVerlag, Berlin, 1993. MR 1202431

[8] K. Konieczna and P. Urbański, Double vector bundles and duality, Arch. Math. (Brno) 35 (1999), no. 1, 59-95. MR 1684522

[9] M. Kureš, Weil modules and gauge bundles, Acta Math. Sin. (Engl. Ser.) 22 (2006), no. 1, 271-278. MR 2200783

[10] M. Kureš and W. M. Mikulski, Liftings of linear vector fields to product preserving gauge bundle functors on vector bundles, Lobachevskii J. Math. 12 (2003), 51-61. MR 1974543

[11] K. C. H. Mackenzie, General Theory of Lie Groupoids and Lie Algebroids, London Mathematical Society Lecture Note Series, 213, Cambridge University Press, Cambridge, 2005. MR 2157566

[12] W. M. Mikulski, Product preserving bundle functors on fibered manifolds, Arch. Math. (Brno) 32 (1996), no. 4, 307-316. MR 1441401

[13] W. M. Mikulski, Product preserving gauge bundle functors on vector bundles, Colloq. Math. 90 (2001), no. 2, 277-285. MR 1876848

[14] W. M. Mikulski, Lifting double linear vector fields to Weil like functors on double vector bundles, Math. Nachr. 292 (2019), no. 9, 2092-2100. MR 4009348

[15] W. M. Mikulski, Prolongation of linear semibasic tangent valued forms to product preserving gauge bundles of vector bundles, Extracta Math. 21 (2006), no. 3, 273-286. MR 2332075

[16] W. M. Mikulski and J. M. Tomáš, Product preserving bundle functors on fibered fibered manifolds, Colloq. Math. 96 (2003), no. 1, 17-26. MR 2013706 
[17] V. V. Shurygin, jr., Product preserving bundle functors on multifibered and multifoliate manifolds, Lobachevskii J. Math. 26 (2007), 107-123. MR 2396705

[18] L. B. Smolyakova and V. V. Shurygin, Lifts of geometric objects to the Weil bundle $T^{\mu} M$ of a foliated manifold defined by an epimorphism $\mu$ of Weil algebras, Russian Math. (Iz. VUZ) 51 (2007), no. 10, 76-88. MR 2381929

Wtodzimierz M. Mikulski

Faculty of Mathematics and Computer Science UJ, ul. Łojasiewicza 6, 30-348, Cracow, Poland Wlodzimierz.Mikulski@im.uj.edu.pl

Received: May 8, 2019

Accepted: June 16, 2020 